\title{
Single-Phase Filamentary Cellular Breakdown Via Laser-Induced Solute Segregation
}

\section{Citation}

Akey, Austin J., Daniel Recht, James S. Williams, Michael J. Aziz, and Tonio Buonassisi. 2015.

“Single-Phase Filamentary Cellular Breakdown Via Laser-Induced Solute Segregation."

Advanced Functional Materials 25 (29) (June 18): 4642-4649. doi:10.1002/adfm.201501450.

\section{Published Version}

doi:10.1002/adfm.201501450

\section{Permanent link}

http://nrs.harvard.edu/urn-3:HUL.InstRepos:34325475

\section{Terms of Use}

This article was downloaded from Harvard University's DASH repository, and is made available under the terms and conditions applicable to Open Access Policy Articles, as set forth at http:// nrs.harvard.edu/urn-3:HUL.InstRepos:dash.current.terms-of-use\#OAP

\section{Share Your Story}

The Harvard community has made this article openly available.

Please share how this access benefits you. Submit a story.

Accessibility 
Advanced Functional Materials, in press (2015).

WILEY-VCH

Article type: Full Paper

DOI: $10.1002 /$

\title{
Single-Phase Filamentary Cellular Breakdown via Laser-Induced Solute Segregation
}

Austin J. Akey*, Daniel Recht, J.S. Williams, Michael J. Aziz, and Tonio Buonassisi

Dr. A. J. Akey, Professor T. Buonassisi

Massachusetts Institute of Technology

77 Massachusetts Avenue Room 35-214

Cambridge, MA 02139, USA

Email: akey@mit.edu

Phone: +1 (518) 588-1660

Dr. D. Recht, Professor M.J. Aziz

Harvard University

204A Pierce Hall, 29 Oxford Street

Cambridge, MA 02138, USA

Professor J.S. Williams

The Australian National University

Cockcroft 4 39, Acton ACT 2601, Australia

Keywords: alloys, microstructures, semiconductors, structure-property relationships

\begin{abstract}
Nanosecond melting and quenching of materials offers a pathway to novel structures with unusual properties. Impurity-rich silicon processed using nanosecond-pulsed-laser-melting is known to produce nanoscale features in a process referred to as "cellular breakdown" due to destabilization of the planar liquid/solid interface. Here, we apply atom probe tomography combined with electron microscopy to show that the morphology of cellular breakdown in these materials is significantly more complex than previously documented. We observe breakdown into a complex, branching filamentary structure topped by a few nm of a cell-like layer. Singlephase diamond cubic silicon highly supersaturated with at least $10 \%$ atomic Co and no detectable silicides is reported within these filaments. In addition, the unprecedented spatio-chemical accuracy of the atom probe allows us to investigate nanosecond formation dynamics of this
\end{abstract}




\section{WILEY-VCH}

complex material. Previously-reported properties of these materials can now be reconsidered in light of their true composition, and this class of inhomogeneous metastable alloys in silicon can be explored with confidence.

\section{Introduction}

In pursuit of novel materials with advanced functionality, non-equilibrium fabrication processes have attracted significant attention ${ }^{[1-5]}$, giving access to a range of structures. However, the shorter length-scales characteristic of some non-equilibrium materials have made it challenging to characterize these systems with existing techniques. With the advent of higherresolution and higher-sensitivity metrology techniques, it has become possible to revisit longstanding problems in materials research, particularly problems of nanoscale structure and composition. This affords the materials research community the opportunity to re-examine processes that may prove more complex than are suggested by low-resolution measurements and models based on them, and to develop more nuanced models of kinetically-determined systems.

High-rate quenching of solid solutions accessible via laser-based processing has been

known for decades ${ }^{[6,7]}$, yet the experimental tools to examine the resulting materials with atomic precision have been lacking. Conventional tools including scanning and transmission electron microscopy (SEM and TEM), and secondary-ion mass spectroscopy (SIMS) can yield surface morphology, cross-sectional morphology, crystallographic structure, and micron-scale composition, but cannot conclusively determine the 3D composition profile with sufficient resolution for full characterization of these materials.

Herein, we apply three-dimensional atom probe tomography (APT) to crystalline silicon that has been ion-implanted with ${ }^{59} \mathrm{Co}$ to a dose of $5 \times 10^{15}$ atoms $/ \mathrm{cm}^{2}$ and then rapidly melted 


\section{WILEY-VCH}

and resolidified by a ns-pulsed UV excimer laser ${ }^{[4]}$. This type of material is known to form a distinctive pattern of segregated material within the laser-melted region, referred to as cellular breakdown ${ }^{[8,9]}$ (due to the presence of surface features resembling cell walls in plants), but the local composition and morphology has not been well characterized. The use of APT to reveal three-dimensional morphology and composition goes well beyond what is possible using TEM and SEM, enabling the first fully three-dimensional mapping of this material. We observe branching filamentary structures of segregated impurities in supersaturated solid solutions, but without formation of secondary crystal phases. Preserving the single-crystalline substrate structure throughout implies local impurity concentrations orders of magnitude beyond the solid solubility limit. While super-saturation of low-solubility transition metals have been observed in homogeneously-mixed specimens ${ }^{[4,5]}$, the local concentrations of Co achieved within the structures observed here are the highest known for any transition metal in single-crystalline silicon, by at least two orders of magnitude ${ }^{[4]}$. We use the APT dataset to investigate the dynamics of the cellular breakdown process, placing a lower limit on the difference in melt duration between the filaments and the rest of the melted layer, and drawing comparisons to steady-state breakdown simulations in the literature. 


\section{WILEY-VCH}

$a$
$\sim 140 \mathrm{~nm} \mid$

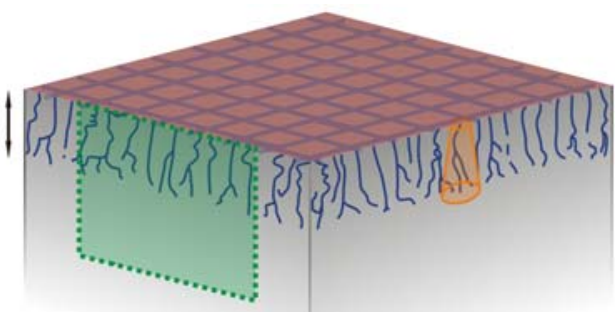

b

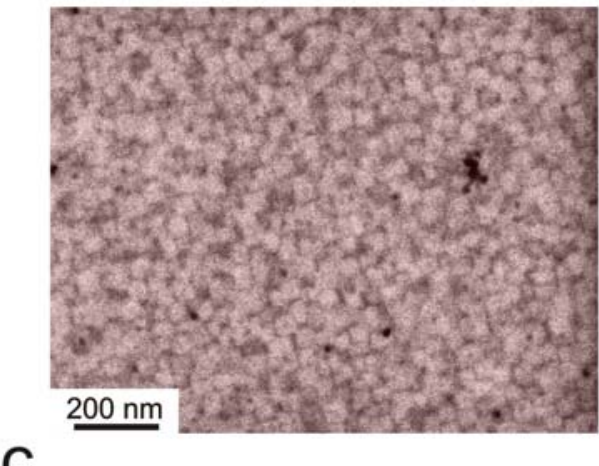

C
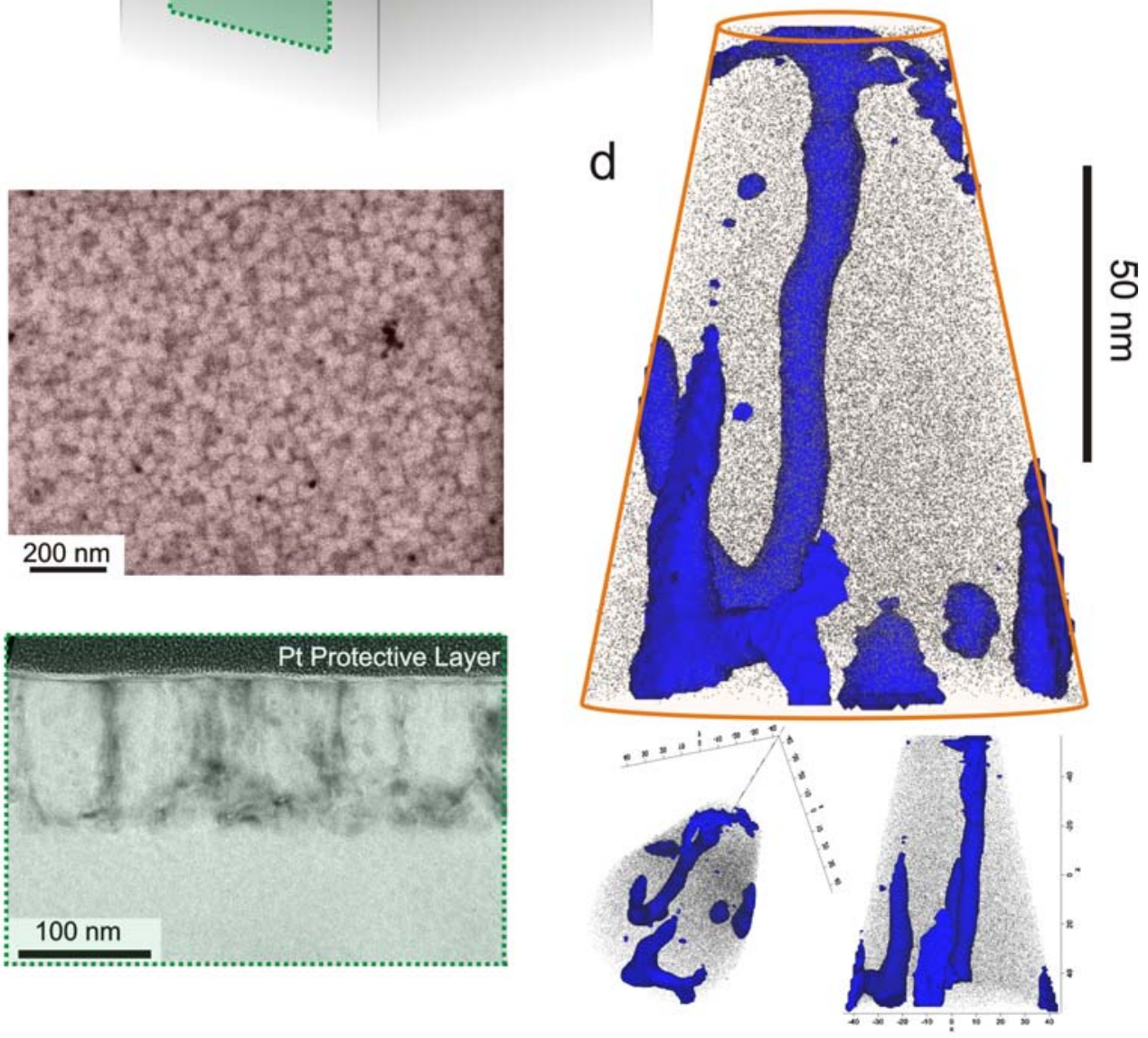

Figure 1. a) Schematic (not to scale) of cobalt-in-silicon cellular-breakdown material. Colored regions correspond to the analysis fields of sections b-d. b) Plan view SEM of cellularbreakdown material surface. c) Bright-field cross-sectional TEM showing cellular breakdown layer above single-crystalline Si. d) APT of cellular breakdown material. Grey dots denote $\mathrm{Si}$ atoms ( $1 \%$ shown, for clarity), while blue surface is a $1 \%$ at. Co concentration isosurface. The capping material above the original wafer surface is omitted. Lower images, to highlight the three-dimensional morphology: at left, a view of the reconstruction in the larger image from an upper oblique angle; at right, a view from the right side of the larger image. 


\section{WILEY-VCH}

\section{Results and Discussion}

\subsection{Structural Characterization}

Plan-view SEM and cross-sectional TEM results closely match those reported in previous publications for this type of material ${ }^{[4]}$; Figure 1a provides an overview of the measurements. In Figure 1b, the classical surface morphology of cellular breakdown can be seen in plan-view SEM (PVSEM), in a pattern of square outlines - assumed to be segregated dopant-rich regions - on the surface. Figure 1c shows a cross-sectional TEM view of this material, with the characteristic vertical, higher-contrast regions that have been identified as "cell walls", ${ }^{\prime 9]}$, usually assumed to be extended two-dimensional structures viewed edge-on in cross-section. In both measurements the spacing of the cellular features is approximately $50 \mathrm{~nm}$. Selective-area electron diffraction

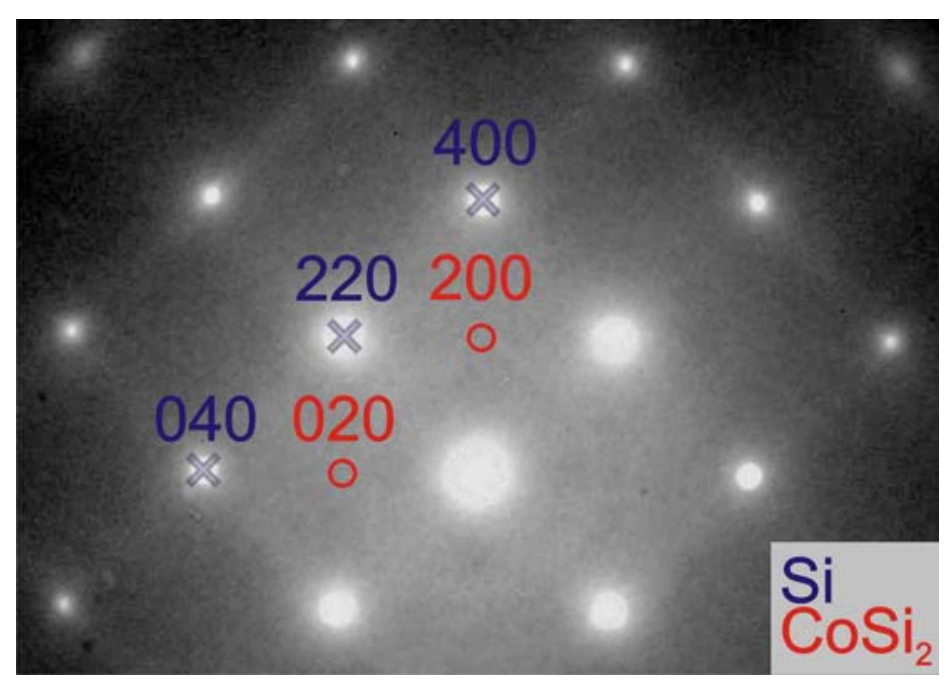

Figure 2. Cross-sectional selective-area electron diffraction taken from the cellular-breakdown region of the specimen. Beam direction was along the [001] axis. Points corresponding to $\mathrm{Si}$ diamond-cubic diffraction are labeled in blue, and marked with crosses; points corresponding to $\mathrm{CoSi}_{2}\left(\mathrm{CaF}_{2}\right.$-type structure) are labeled in red, marked with open circles. No evidence of $\mathrm{CoSi}_{2}$ structure is observed. 


\section{WILEY-VCH}

(SAED, Figure 2) from this layer shows no evidence of the known cobalt silicides, either stable or metastable. There is also no evidence of polycrystalline material.

Figure 3 shows the Secondary Ion Mass Spectrometry (SIMS) concentration vs depth

analysis of ${ }^{59} \mathrm{Co}$ content in this material, again closely matching that reported in previous literature, including the peak in concentration at a depth of $110 \mathrm{~nm}$ which is believed to represent the depth at which the onset of cellular breakdown occurred. Figure 3 also contains the APTderived concentration vs depth profile for ${ }^{59} \mathrm{Co}$ in this material. Despite the fact that APT samples many orders of magnitude fewer atoms than SIMS, it can be seen that the two measurements yield comparable results for concentration vs depth, with some excursions in the APT measurement (here APT is providing a fine-grained view of a much smaller volume than SIMS).

Figure 1d shows the three-dimensional APT reconstruction of material that has undergone morphological breakdown. From this reconstruction, it is evident that the highimpurity regions are not two-dimensional "cell walls", but rather filamentary structures extending upward toward the surface (see also Animation S1 in the Supporting Information). These structures average approximately $8 \mathrm{~nm}$ in diameter. At the surface, the deeper filamentary segregation region transitions to a thin (approximately $3 \mathrm{~nm}$ in depth) in-plane stripe of impurities, which appears to correspond to the square outlines observed in plan-view microscopy. There are several isolated regions of high impurity concentration below the surface, 


\section{WILEY-VCH}

disconnected from the larger filaments.

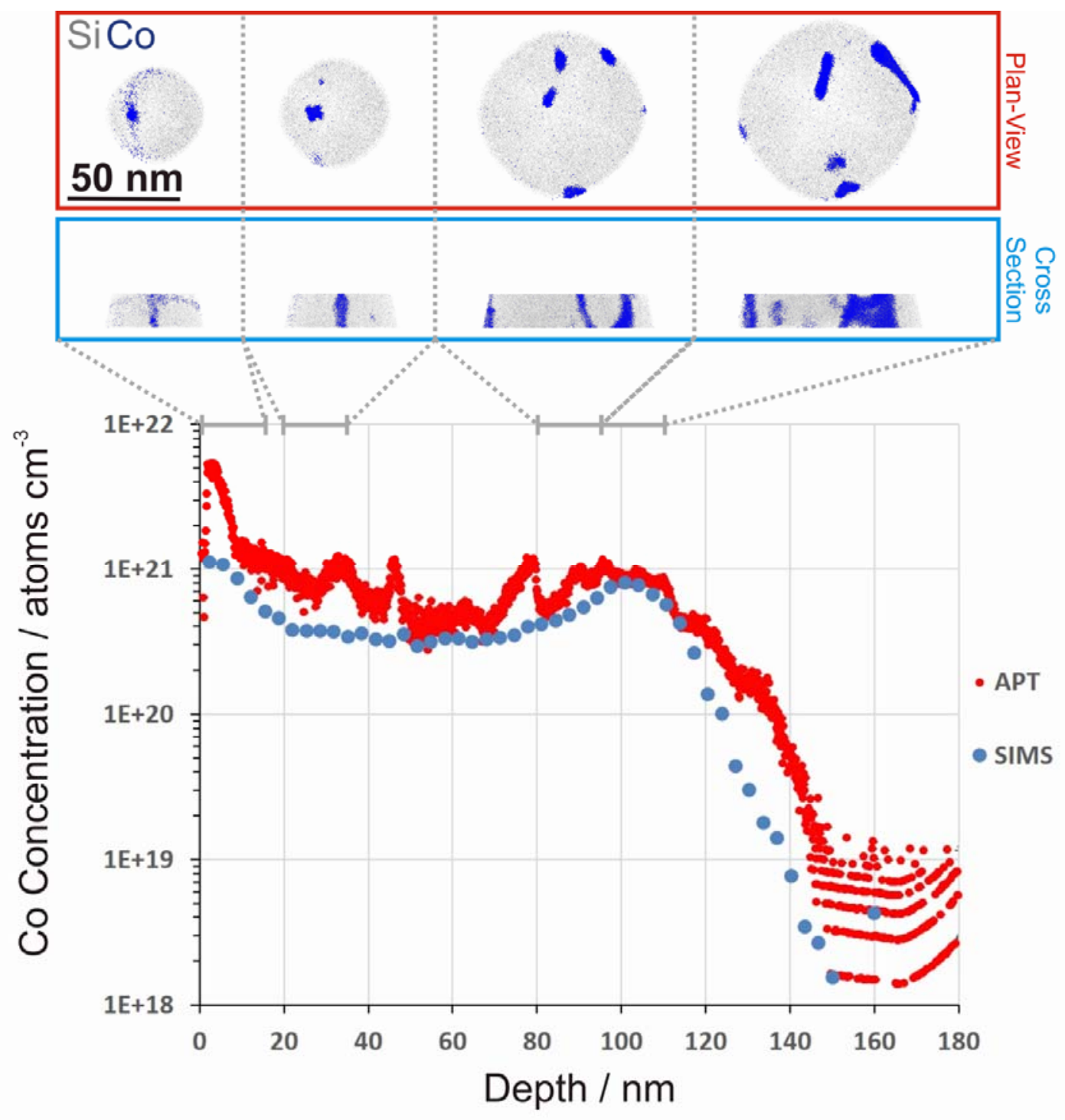

Figure 3. Above, $15 \mathrm{~nm}$-thick slices of the corresponding APT dataset, side (blue box) and top (red box) views, with grey dots representing Si atoms (1\% shown for clarity) and blue dots representing Co atoms (100\% shown). Below, APT and SIMS 1D concentration vs depth profiles. Grey marker bars at the top denote the corresponding depth range of each displayed APT slice. The APT depth profile has been scaled starting from the original wafer surface, as determined by the position of the wafer's native Si oxide layer. 


\section{WILEY-VCH}

It should also be noted that the area that would correspond to the "inside" of the squares seen on the surface is not free of Co at all depths; some areas have only a few individual Co atoms, while others contain branches of the Co-rich filaments. Cross sections from the APT dataset can be seen in Figure 3, providing a view of the local morphology of the segregated regions at various depths. Finally, the transition to morphological breakdown is clear and abrupt, with segregated regions appearing discontinuously above the region of breakdown initiation (See also Figure S1) without any disconnected segregation regions below the filaments.

An analysis of the impurity content of the segregation filaments can be seen in Figure 4 . Impurity concentration increases toward the center of the segregation filaments, with some areas having Co content above $10 \%$ (atomic \%). Co content varies both with depth and from the edge to the center of the filaments, with the highest concentrations appearing in small regions at branching points where two filaments connect. The lateral Co concentration distribution through the filaments is challenging to determine accurately, due to experimental artifacts. There is a known artifact during APT field evaporation that arises from two linked sources: the change in dielectric constants in regions of high doping, and the evolution of a locally different radius of curvature due to differing evaporation fields, both causing lateral deviation of electric field lines

from the surface-normals assumed during data reconstruction ${ }^{[10-13]}$. This causes areas of differing composition to be "magnified" in the reconstructed data. From the literature ${ }^{[14,15]}$, it is known that ion trajectory overlaps near the edges of precipitates can lead to incorrect absolute concentration measurements in these regions. However, for the centers of higher-field impurity regions larger than $2 \mathrm{~nm}$ this issue is believed to be negligible, and is not expected to significantly affect the atomic-percent-scale compositions reported here except within the outermost $1 \mathrm{~nm}$ of the filament where the measured composition is likely too low. 


\section{WILEY-VCH}
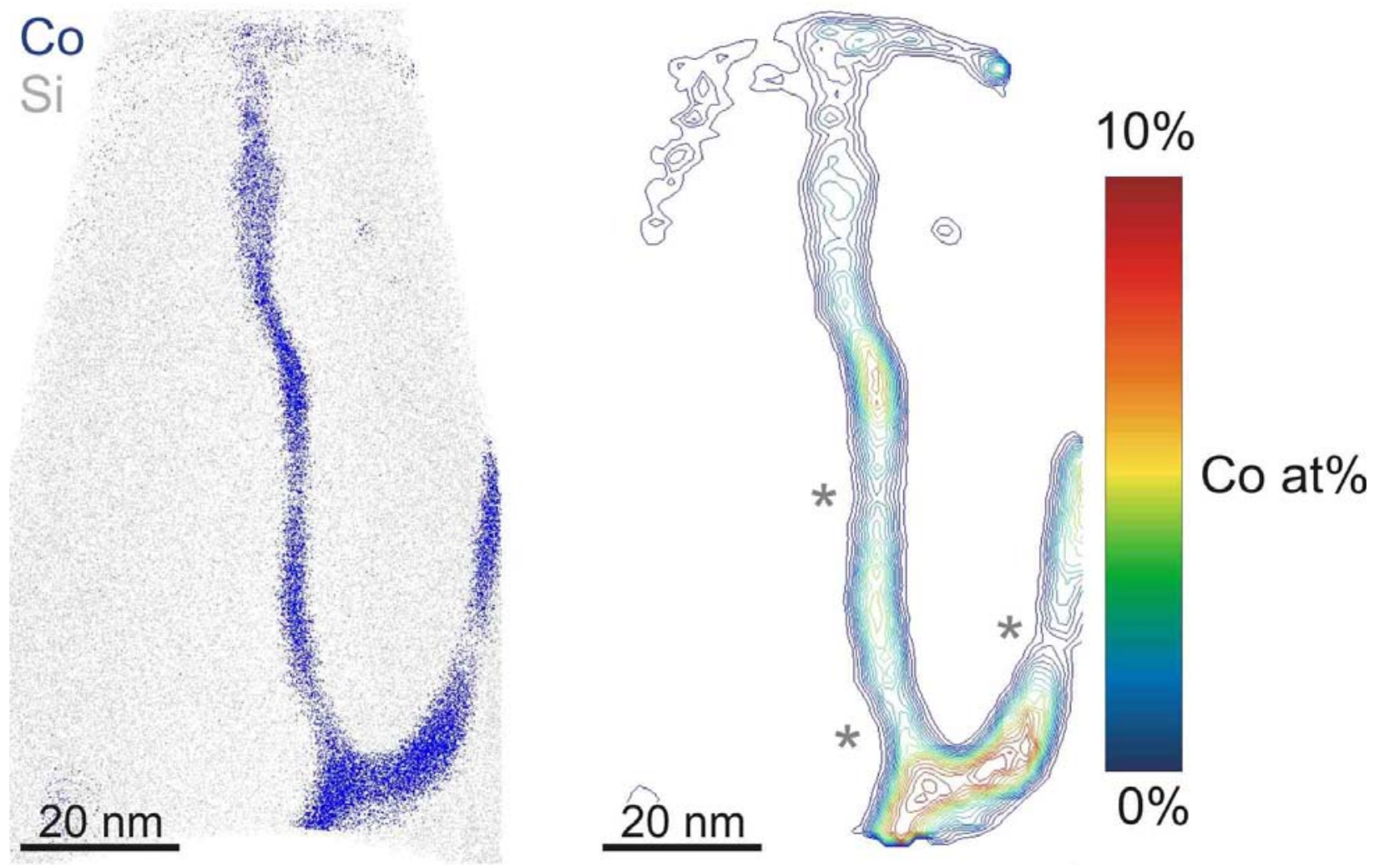

Figure 4. Left, a cross-sectional $15 \mathrm{~nm}$ thick slice from an APT dataset, fully enclosing one filamentary breakdown region. Grey atoms denote $\mathrm{Si}$ (1\% shown for clarity) while blue atoms denote Co (100\% shown). A small ( 2 nm) layer of aSi capping material is shown at the top of the image, above the original wafer surface. Right, a 2D Co atomic concentration isocontour plot, with contours in $1 \%$ at. increments. Asterisks indicate regions of filament where the composition gradient qualitatively resembles "pinch-off" morphology.

The issue of preferential field evaporation/retention of specific elements in APT can be addressed by comparing the SIMS and APT one-dimensional concentration profiles, which are in good agreement, indicating that there is insufficient preferential evaporation to invalidate the APT measurement. 


\section{WILEY-VCH}

\subsection{A Novel Inhomogeneous Alloy}

The segregation regions are shown here to contain a stunningly high concentration of Co $(10 \%)$ within crystalline Si. This is two orders of magnitude greater than the highest previously attained concentration of any transition metal, and 5,000 times the prior experimental upper limit on the dissolved cobalt concentration in homogeneous solution in Si. This metastable phase of Co in Si could have qualitatively different optical and electronic properties from lower-impurity Si. In addition, given the similarity of these structures to those observed in other transition-metalimplanted, pulsed-laser-melted Si specimens ${ }^{[4]}$, it is likely that there exists a family of these unexplored metastable Si alloys. Silicon's technological importance and unparalleled degree of scientific development make this discovery of potentially great interest to materials science and semiconductor technology, changing the status of these materials from unexamined and not yet exploited (due to uncertainties about their composition) to materials open for exploration. At present there is no known way to deliberately create large quantities of these impurity-rich phases, nor is there a method for tailored formation at specific sites, but now that it is possible to measure the results of such efforts, real progress can be made. Previous results that report interesting properties in cellularly-broken-down specimens can now be re-examined from a position of better understanding ${ }^{[16,17]}$.

It is important to consider the crystalline nature of the segregation regions, both for understanding the formation process and for any attempts at modeling the material's properties. Metastable phases exist of a wide variety of transition metal silicides ${ }^{[18,19]}$, but the SAED data in Figure 2 shows no evidence of the lowest-Si-content cobalt silicide, $\mathrm{CoSi}_{2}$, which has the fluorite crystal structure and would be easily distinguished from diamond cubic. There is also no polycrystalline material signal in the diffraction data, which implies that the entire breakdown 


\section{WILEY-VCH}

region is monocrystalline; amorphous material is not expected to form during PLM in this interfacial-velocity regime due to explosive-crystallization effects ${ }^{[20]}$, and no evidence has ever been seen of amorphous ultrafast cellular-breakdown material (including in channeling Rutherford Backscattering measurements ${ }^{[21]}$ and in UV Raman ${ }^{[22]}$ analysis of similar materials). While the position of the Co atoms within the diamond-cubic Si lattice cannot be directly deduced from the data available, one possibility would draw an analogy between $\mathrm{CoSi}_{2}$ formation here and $\gamma-\mathrm{FeSi}_{2}$ platelets, which are known to be lattice-matched to surrounding $\mathrm{Si}$ crystal due to interface energy ${ }^{[23]}$. However, even with only one third of the Co sites filled, the shift of the Si positional symmetry from the diamond-cubic to the fluorite unit cell would still result in diffraction spots that should be detectable by SAED; thus, it is very unlikely that any significant fraction of the material is composed of silicide structures.

\subsection{Decomposition of Dataset}

Lateral segregation during solidification moves a large amount of Co into the filament regions, but there is still a super-saturation of Co in the rest of the solidified layer. By creating a $1 \%$ Co concentration isosurface, we divide the analyzed volume into two datasets: one that contains only the segregated regions, defined here as those regions with greater than $1 \%$ Co and encompassing all of the filamentary structures, and those regions with less than $1 \% \mathrm{Co}$, which are featureless and homogeneous. A concentration vs depth profile of these two regions (Figure 5) shows that the segregated Co component distribution does not match the SIMS data, and in fact is relatively constant with depth, implying that the Co distribution within these regions follows a different 


\section{WILEY-VCH}

mechanism from the one active outside of the segregated areas.

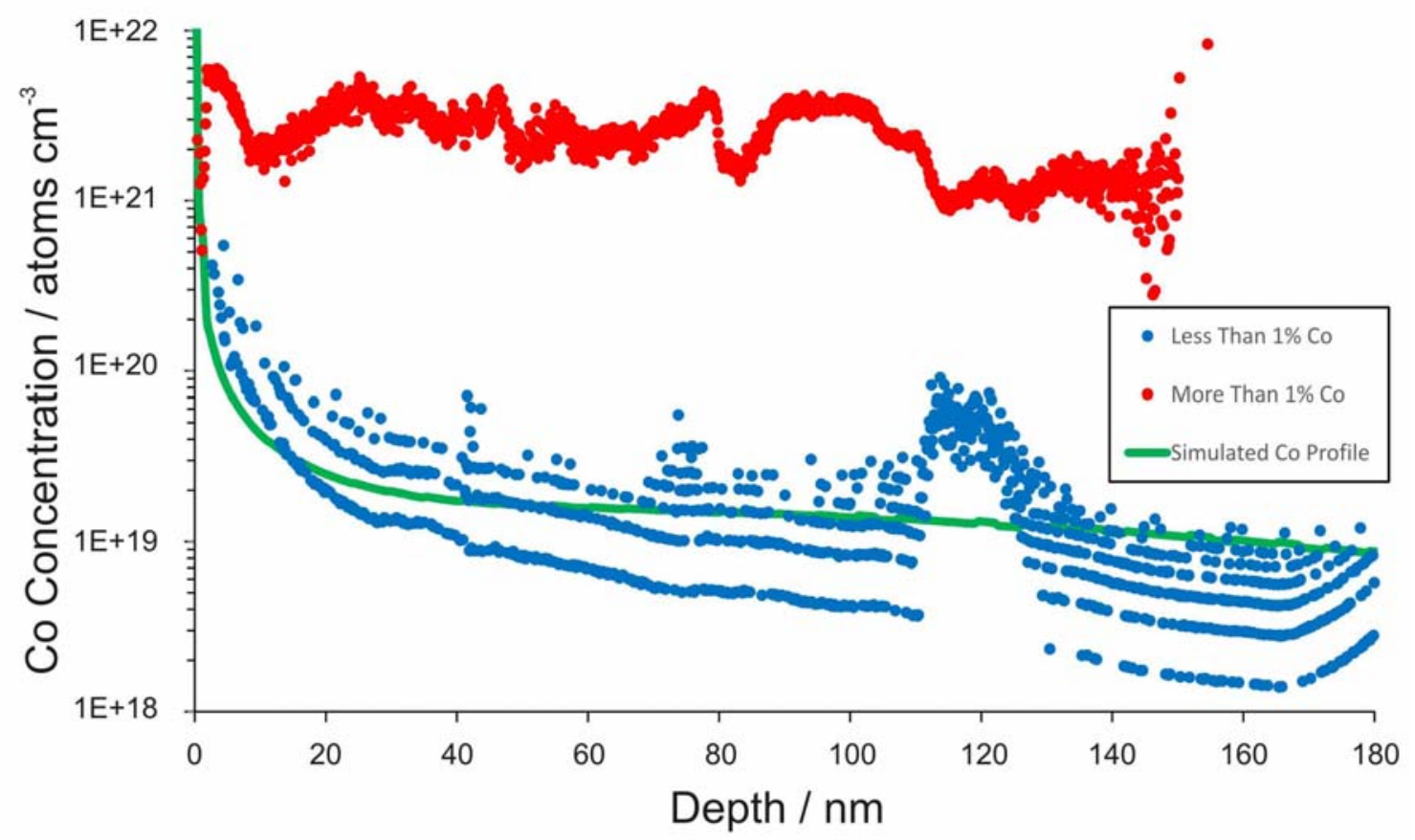

Figure 5. 1D composition vs depth profiles of the decomposed APT dataset. In red, the portion of the data enclosed by a $1 \%$ atomic Co concentration isosurface. In blue, the remaining dataset outside of that surface (not including the capping material). In green, a simulation as described in $\operatorname{Ref}^{[24]}$ in the main text, with a one-parameter fit to the blue curve. Note that the y-axis striation of the blue curve is the result of single-Co-atom differences within the thin z-sections used to calculate this profile.

The impurity-rich regions in PLM cellular breakdown contain an average Co concentration of around $6 \%$. The non-segregation regions, by contrast, are also plotted in Figure 5, showing a distribution more characteristic of ion-implanted, PLM-treated materials such as Au in Si where no cellular breakdown has occurred ${ }^{[5]}$. Following the same fitting procedure used in ${ }^{[4,24,25]}$, we can fit the redistribution of the Co in these regions during the melting process, using 


\section{WILEY-VCH}

the diffusivity of Co in both solid ${ }^{[26,27]}$ and molten ${ }^{[28]} \mathrm{Si}$, as well as the measured equilibrium partition coefficient of Co in Si from ${ }^{[29]}, k_{\mathrm{e}}=9 \times 10^{-4}$. We find that the best fit to the near-surface region is given by a diffusive velocity of approximately $400 \mathrm{~m} / \mathrm{s}$, close to the value of $350 \mathrm{~m} / \mathrm{s}$ seen for $\mathrm{Au}$ in $\mathrm{Si}^{[5]}$, and much less than the value of $10^{4} \mathrm{~m} / \mathrm{s}$ previously assumed for these materials ${ }^{[4]}$ (which came from assuming that the Co concentration peaks observed at $110 \mathrm{~nm}$ and at the surface in SIMS measurements were due entirely to segregated species). This type of analysis should enable more accurate evaluation of the diffusive velocity of transition metals during solidification, without the confounding effects of breakdown, and also allows the breakdown process itself to be further investigated.

\subsection{Cellular Breakdown Dynamics}

The three-dimensional morphological complexity of this material is considerably greater than a simple one-dimensional interface model can reflect; however, it is possible to make useful statements about the breakdown process without going to a full three-dimensional model such as

a phase-field simulation ${ }^{[30]}$. It is worth noting that such simulations have assumed steady-state solidification of dilute binary alloys, whereas the cellular breakdown process we have studied represents the initiation of breakdown and a brief propagation period, and is unlikely to have reached steady state. Given this difference, it is interesting to note that our observations bear some similarities to the breakdown structures predicted in Ma and Plapp ${ }^{[30]}$, including both the presence of filaments and the bridges between them at the surface, but with filamentary branching and without the "droplets" of impurity-enriched material at the bottom of the simulated breakdown zone, which will be discussed later. 


\section{WILEY-VCH}

The critical questions in this process revolve around the movement of impurity atoms on the nanosecond and nanometer scale. The standard model of cellular breakdown during pulsedlaser melting invokes the formation of lateral (i.e., parallel to the solid-liquid interface) variations in impurity concentration within the melt ${ }^{[31-34]}$. The more impurity-rich regions serve to locally retard the progress of the solidification front through the mechanism of constitutional supercooling, leading to "troughs" of molten, impurity-rich material surrounded by solid crystal. A phenomenological treatment of the initial transient of melt-front destabilization can be found in $^{[35]}$. In principle the local impurity concentration should determine the degree of melting-point depression, and thus the duration of the molten phase in these segregation regions. Segregation of impurities at the solid/liquid interface in these molten "troughs" may continue for some time after the major solidification front has moved upward toward the original wafer surface, causing the amount of impurity contained within the still-molten regions to continue increasing until it either equilibrates compositionally or the liquid finally cools enough to completely solidify. Estimating the duration of this process it thus critical to understanding the material's formation.

We use the measured final morphology and composition of the filaments to elucidate their formation process. We distinguish between three types of impurity movement within the material: i) redistribution within the molten material, ii) diffusion within the solid, and iii) segregation at the solid/liquid interface. We can safely discount the first mechanism outside of the filaments, as there is no miscibility gap on the dilute $\mathrm{Co}$ side of the $\mathrm{Co} / \mathrm{Si}$ binary phase diagram $^{[36]}$, and thus there will not be diffusion "up" the concentration gradient within the melt. Equally, there should be no special diffusivity along the solid/liquid interface due to the higher mobility in the melt. The third mechanism is well understood in this system, as will be seen below. Bulk diffusion in the solid, as well as diffusion in the confined regions that become the 


\section{WILEY-VCH}

observed filaments, requires a more in-depth treatment. Whereas the initial heating to $\sim 4000 \mathrm{~K}$ takes place over the duration of the nanosecond laser pulse, the cooling rate is much slower, being determined by the thermal conductivity of the sample. As a result, areas of the melted and solidified film experience elevated temperature for on the order of a hundred nanoseconds after solidification, making it possible for impurities to diffuse through the crystal toward sinks such as surfaces and interfaces. Given this high-diffusivity interval, it is interesting to ask both why the Co in these samples, which is known to be a fast diffuser in $\mathrm{Si}^{[27]}$, has not migrated downward from the free surface, and whether the final shapes of the filaments are purely representative of the perturbations in the solidification front, or are instead modified by diffusion following solidification.

The high diffusivity and low enthalpy of migration of $\mathrm{Co}$ in $\mathrm{Si}^{[27]}$, would allow Co to diffuse on the order of hundreds of nm (see the Supporting Information) despite the rapid cooling in pulsed-laser-melting. This is much larger than the observed filaments, and thus requires explanation. Density functional theory predicts that while the Co interstitial defect in Si is highly mobile at these temperatures, the Co interstitial dimer (two Co interstitials in proximate sites) is immobile due to its high binding energy ${ }^{[37]}$. At the concentrations reached in the measured material, it is possible that most or all of the Co atoms are present as dimers and higher-order interstitial defects, locking the solidified material into its observed configuration.

It remains to address the question of diffusion and segregation of the impurities into and throughout the filamentary regions. We assume that the depression of the Si freezing point with Co content means that the impurity-rich filament material remains molten after the solidification of the impurity-poor non-filamentary region at the same depth. As this system is far from equilibrium, we estimate the kinetic liquidus temperature for both regions using the Continuous 


\section{WILEY-VCH}

Growth Model of solute trapping ${ }^{[38]}$. We neglect here the influence of crystalline orientation on the interfacial undercooling, treating all solidification as occurring along the [100] direction, such that the equilibrium liquidus temperature will act as an upper bound for all ${ }^{[39]}$.

First, we calculate $k(v)$ the kinetically-determined partition coefficient as a function of the solidification velocity $v$, estimated using the earlier simulations of the pulsed-laser-melting to be $3.5 \mathrm{~m} / \mathrm{s}$, using also the diffusive velocity of $\mathrm{Co}$ in $\mathrm{Si}$ (measured from the above fits to the decomposed dataset), $v_{D}=400 \mathrm{~m} / \mathrm{s}$, and the equilibrium partition coefficient ${ }^{[29]} k_{e}:=9 \times 10^{-4}$ :

$$
k(v) \equiv \frac{X_{S}}{X_{L}}=\frac{k_{e}+\frac{v}{v_{D}}}{1+\frac{v}{v_{D}}}
$$

where $X_{S}$ and $X_{L}$ are the impurity composition of the solid and liquid, respectively, at the interface ${ }^{[38]}$. For $v=3.5 \mathrm{~m} / \mathrm{s}, k=9.6 \times 10^{-3}$.

Next, using $m_{L}^{e}$ the slope of the equilibrium liquidus from the dilute Co side of the equilibrium Co-Si binary phase diagram ${ }^{[36]}, m_{L}^{e}=6.89 \mathrm{~K} / \mathrm{a} / \mathrm{Co}$, we calculate the slope of the kinetic liquidus:

$$
m_{L}(v)=m_{L}^{e}\left(\frac{1-k+[k+(1-k) \beta] \ln \left(\frac{k}{k_{e}}\right)}{1-k_{e}}\right)
$$

Here $\beta$ is a term related to the presence or absence of solute drag; for this system we assume no solute drag, in accordance with the only experimental measurement ${ }^{[40]}$, and set $\beta=0$. In the dilute limit, using the above values we find the kinetic liquidus slope to be $m_{L}=6.98 \mathrm{~K} / \mathrm{a} / \mathrm{C}$.

We can now use the time-temperature profile of the sample to investigate the dynamics of solidification in the filaments. Here we estimate the time delay for solidification between nonfilament and filament regions. See the Supporting Information (Figure S2) for simulated temperature vs. time and depth profiles. From these profiles, we determine that the temperature 


\section{WILEY-VCH}

gradient in depth is approximately $0.6 \mathrm{~K} / \mathrm{nm}$, with an average solidification interface velocity of $3.5 \mathrm{~m} / \mathrm{s}$, yielding a cooling rate in the layer of interest of approximately $2.1 \mathrm{~K} / \mathrm{ns}$. The final piece of information needed is the composition of the molten liquid at the liquid/solid interface in the non-filament regions. We can find this composition using the decomposed dataset in Figure 5; the average composition of the solidified non-filament regions, disregarding the peak at the free surface, is found to be $\sim 1.4 \times 10^{19} \mathrm{Co}$ atoms $/ \mathrm{cm}^{3}$. Using Eq. 1, we calculate the kinetic partition coefficient at the interface to be $9.6 \times 10^{-3}$, meaning that the composition of the liquid at the interface in the non-segregation regions was $\sim 1.5 \times 10^{21} \mathrm{Co}$ atoms $/ \mathrm{cm}^{3}$, or $2.9 \% \mathrm{Co}$.

Using the slope of the kinetic liquidus, we calculate the difference in liquidus temperature between the non-filament and filament regions, using $10 \%$ Co as an estimated lower limit of the composition of the liquid in the filament regions at the instant of solidification. The difference in kinetic liquidus temperature is calculated to be $49 \mathrm{~K}$, and thus the filament regions solidify at least $23 \mathrm{~ns}$ after the non-filament regions. Note that this is a lower limit on the time delay, as it derives from a difference in liquidus temperatures; the difference between solidus temperatures is likely equal or greater, leading to a correspondingly longer time delay, especially with crystalline anisotropy taken into account. This is the time available for lateral segregation from solid non-filament areas to the still-molten filaments. Quantitative evaluation of the amount of interface segregation vs bulk diffusion of Co within the molten filaments would require a full three-dimensional model, which we do not attempt here.

Finally, we address the difference between the morphology observed here and the simulations of $\mathrm{Ma}$ et $\mathrm{al}^{[30]}$. While the filaments and their transition, near the surface, to planar "cells" match the phase-field simulations qualitatively, the filaments are not divided into discrete "droplets" deeper within the specimen, and the filaments display branching behavior at multiple 


\section{WILEY-VCH}

depths (see also Supporting Information Figure S2). We also note the observed square surfacecell morphology, which differs from the hexagons seen in the reported simulation, and is presumably due to crystallographic anisotropy in the interfacial tension. In addition there is an absence, in our measurements, of impurity enrichment in the "cell wall" regions beneath the surface layer which are characteristic of steady-state cellular solidification.

A logical test of the phase-field theory would be to calculate the so-called "pinch-off time" for the filaments here, that is, the time it takes for a Rayleigh instability in the filament to amplify and cause the filament diameter to reduce to zero locally ${ }^{[41]}$. This class of topological singularity has been the subject of recent theoretical interest ${ }^{[42]}$, and a framework has been developed allowing pinch-off to be predicted, verified through measurements of the $\mu \mathrm{m}$-scale evolution of dilute binary alloys using x-ray tomography ${ }^{[43]}$. Given the lack of discrete droplets at the bottom of the observed filaments, we expect the calculated pinch-off time to exceed the calculated melt duration. Unfortunately, as detailed in the Supporting Information, the Coenriched filaments we observe are sufficiently small that the quasi-stationary approximation underpinning the pinch-off calculations is invalid, and so the existing theory cannot be used to explain our observations. If naively applied it yields a pinch-off time of $3.5 \mathrm{~ns}$, which if true would certainly lead to droplet formation. It is interesting to note, however, as seen in Figure 4, that within the filaments there exist several regions in which the Co concentration takes the form of elongated regions of high impurity concentration separated by short regions where the concentration diminishes both axially and radially (indicated by asterisks). We speculate that this may be indicative of a pinch-off mechanism similar to that invoked above, mediated by bulk diffusion of the impurity within the molten filament, but with a different characteristic timescale, 


\section{WILEY-VCH}

one long enough that pinch-off was not completed during the $\geq 23 \mathrm{~ns}$ extra solidification time in the filaments.

\section{Conclusions}

The 3D structure and composition of Si cellular-breakdown material formed by Co ion implantation and pulsed-laser melting are found to be composed of branching impurity-rich filaments. Impurity concentrations within the segregation regions are shown to reach at least 10 $\%$ in some areas, which is two orders of magnitude more Co than has previously been reported in diamond-cubic $\mathrm{Si}$, and more than has previously been reported for any transition metal. Even the non-segregated regions contain Co concentrations orders of magnitude above the equilibrium solid solubility limit. With the spatio-chemical information provided by atom probe tomography, the nanosecond formation dynamics are investigated, and the groundwork laid for a full description of ultrafast cellular breakdown. Comparison with existing steady-state breakdown simulation finds points of agreement and disagreement, the latter possibly arising from the transient nature of this process. Further development of this remarkable class of semiconductor/metal alloy will require improved fabrication methods to control the quantity and location of the impurity-rich material, supported by an extension of cellular solidification existing theory to the non-steady-state. Previously reported properties of these materials can now be reconsidered from a position of greater understanding,

\section{Experimental Section}

Samples of cellular-breakdown material were prepared from wafers of single-crystalline Si ((100), p-type boron doping, $10 \Omega$-cm) by ion implantation followed by pulsed laser melting, as described in Ref. ${ }^{[4]} .{ }^{59} \mathrm{Co}^{+}$was ion-implanted at $120 \mathrm{keV}$ to a dose of $5 \times 10^{15}$ atoms $/ \mathrm{cm}^{2}$ at an 


\section{WILEY-VCH}

angle of 7 degrees from normal, with the substrate held at 77 K (see Figure S5 in the Supporting Information for the as-implanted Co depth profile). Co was chosen for ease of APT analysis, as multiply-charged ${ }^{59} \mathrm{Co}$ ions are not degenerate with any $\mathrm{Si}$ isotopes, and for relevance to both the older literature and recent published results ${ }^{[1,44]}$. Pulsed laser melting was performed using a 308 $\mathrm{nm} \mathrm{XeCl} \mathrm{excimer,} 25 \mathrm{~ns}$ FWHM pulse, with a single pulse of $1.7 \mathrm{~J} / \mathrm{cm}^{2}$ fluence, with an estimated melt depth of $350 \mathrm{~nm}$. All SEM, TEM, SIMS, and APT analyses shown here were carried out on the same piece of implanted material. Plan-view SEM was performed using a ZEISS Ultra-Plus field-emission SEM. TEM samples were prepared via Focused Ion Beam (FIB) cross-sectioning and liftout, using a Zeiss NVision dual-beam Ga FIB fitted with a gasinjection system and an OmniProbe nanomanipulator, operated at $30 \mathrm{kV}$. Further thinning and polishing of the cross-sections was accomplished using a Fischione NanoMill 1040 Ar-ion mill, with an accelerating voltage of $500 \mathrm{eV}$. TEM analysis was performed on a JEOL 2010F HRTEM operated at $200 \mathrm{kV}$. SIMS measurements were performed using a Physical Electronics 6650 Dynamic SIMS instrument with concentration calibrated against the known implant dose in the as-implanted region, and depth measured ex-situ by contact profilometry.

For APT analysis, samples must be mounted on a conductive post and shaped and sharpened into conical needles of $<100 \mathrm{~nm}$ tip radius. In order to allow analysis of the full depth of Co-bearing material, including the surface of the original wafer, the specimens were first coated with a 100-nm layer of Si by electron-beam evaporation, with the substrates held at room temperature by a cooling stage. The specimens were cleaned before deposition with acetone, isopropanol, and deionized water to remove organic contaminants; no corrosive cleaning was performed so as not to disrupt the surface features by removal of the native oxide and subsequent re-oxidation. Samples were then processed into APT-compatible microtips using FIB lift-out, 


\section{WILEY-VCH}

and sharpened by annular ion milling at $5 \mathrm{kV}$. Tips were shaped to contain a thin layer of deposited Si on top of the cellular breakdown material. APT measurements were made in a Cameca LEAP 4000HR operating in laser mode with a 355-nm laser. APT measurement conditions for all specimens were chosen to allow the full depth of the Co-bearing layer to be analyzed, and were as follows: laser repetition rate of $100 \mathrm{kHz}$, laser pulse energy of $40 \mathrm{pJ}$, base temperature of $40 \mathrm{~K}$, and detection rate of $1 \%$. Given the known diffusivity of Co in $\mathrm{Si}$, it is very unlikely that the APT process affected the distribution of Co in the specimen. APT data were reconstructed and analyzed using Cameca Integrated Visualization and Analysis (IVAS) software, with the reconstructions calibrated using the known crystallographic plane spacing of the Si crystal along the $<100>$ direction, as has been described elsewhere ${ }^{[45]}$..

\section{Supporting Information}

Supporting Information is available from the Wiley Online Library or from the author.

\section{Acknowledgements}

The authors thank A. Karma, P.W. Voorhess, W. Kurz, A. Magyar and B. Gault for helpful discussions. This work was primarily supported by Bay Area Photovoltaic Consortium (BAPVC) under contract No. DE-EE0004946. JSW acknowledges the ARC for financial support and access to ANFF ion implantation facilities. 


\section{WILEY-VCH}

[1] Y. Zhou, F. Liu, X. Song, J. Appl. Phys. 2013, 113, 103702.

[2] Y. Zhou, F. Liu, M. Zhu, X. Song, P. Zhang, Appl. Phys. Lett. 2013, 102, 222106.

[3] T. G. Kim, J. M. Warrender, M. J. Aziz, Appl. Phys. Lett. 2006, 88, 241902.

[4] D. Recht, M. J. Smith, S. Charnvanichborikarn, J. T. Sullivan, M. T. Winkler, J. Mathews, J. M. Warrender, T. Buonassisi, J. S. Williams, S. Gradečak, M. J. Aziz, J. Appl. Phys. 2013, 114, 124903.

[5] J. P. Mailoa, A. J. Akey, C. B. Simmons, D. Hutchinson, J. Mathews, J. T. Sullivan, D. Recht, M. T. Winkler, J. S. Williams, J. M. Warrender, P. D. Persans, M. J. Aziz, T. Buonassisi, Nat. Commun. 2014, 5.

[6] J. Krynicki, J. Suski, S. Ugniewski, R. Grotzschel, R. Klabes, U. Kreissig, J. Rudiger, Phys. Lett. A 1977, 61.

[7] A. G. Cullis, H. C. Webber, J. M. Poate, A. L. Simons, Appl. Phys. Lett. 1980, 36, 320.

[8] R. Sriranganathan, D. J. Wollkind, D. B. Oulton, J. Cryst. Growth 1983, 62, 265.

[9] A. G. Cullis, Appl. Phys. Lett. 1981, 38, 642.

[10] V. Gasnier, B. Gault, H. Nako, Y. Aruga, G. Sha, S. P. Ringer, Ultramicroscopy 2013, 132, 199.

[11] F. Vurpillot, B. Gault, B. P. Geiser, D. J. Larson, Ultramicroscopy 2013.

[12] C. Oberdorfer, G. Schmitz, Microsc. Microanal. 2011, 17, 15.

[13] M. K. Miller, M. G. Hetherington, Surf. Sci. 1991, 246, 442.

[14] F. Vurpillot, A. Bostel, D. Blavette, Appl. Phys. Lett. 2000, 76, 3127.

[15] D. Blavette, F. Vurpillot, P. Pareige, A. Menand, Ultramicroscopy 2001, 89, 145.

[16] E. Antolín, A. Martí, J. Olea, D. Pastor, G. González-Díaz, I. Mártil, A. Luque, Appl. Phys. Lett. 2009, 94, 042115.

[17] J. Olea, A. Del Prado, D. Pastor, I. Mártil, G. González-Díaz, J. Appl. Phys. 2011, 109, 113541.

[18] C. M. Comrie, J. Appl. Phys. 2004, 95, 2365.

[19] I. Dézsi, C. Fetzer, I. Szücs, J. Dekoster, A. Vantomme, M. Caymax, Surf. Sci. 2005, 599, 122.

[20] M. O. Thompson, G. J. Galvin, J. W. Mayer, P. S. Peercy, J. M. Poate, D. C. Jacobson, A. G. Cullis, N. G. Chew, Phys. Rev. Lett. 1984, 52, 2306.

[21] J. Narayan, J. Cryst. Growth 1982, 59, 583.

[22] D. Pastor, J. Olea, A. del Prado, E. García-Hemme, I. Mártil, G. González-Díaz, J. Ibáñez, R. Cuscó, L. Artús, Semicond. Sci. Technol. 2011, 26, 115003.

[23] M. G. Grimaldi, G. Franzo, S. Ravesi, A. Terrasi, C. Spinella, A. La Mantia, Appl. Surf. Sci. 1994, 74, 19.

[24] B. P. Bob, A. Kohno, S. Charnvanichborikarn, J. M. Warrender, I. Umezu, M. Tabbal, J. S. Williams, M. J. Aziz, J. Appl. Phys. 2010, 107, 123506.

[25] T. Kim, M. R. Pillai, M. J. Aziz, M. A. Scarpulla, O. D. Dubon, K. M. Yu, J. W. Beeman, M. C. Ridgway, J. Appl. Phys. 2010, 108, 013508.

[26] A. Appelbaum, J. Vac. Sci. Technol. B Microelectron. Nanometer Struct. 1987, 5, 858.

[27] J. Utzig, D. Gilles, Mater. Sci. Forum 1989, 38-41, 729.

[28] K. Tang, E. Ovrelid, G. Tranell, M. Tangstad, JOM 2009, 61, 49.

[29] J. R. Davis, A. Rohatgi, R. Hopkins, P. Blais, P. Rai-Choudhury, J. Mccormick, H. C. Mollenkopf, IEEE Trans. Electron Devices 1980, 27.

[30] Y. Ma, M. Plapp, J. Cryst. Growth 2014, 385, 140.

[31] D. E. Hoglund, M. J. Aziz, S. R. Stiffler, M. O. Thompson, J. Y. Tsao, P. S. Peercy, J. Cryst. Growth 1991, 109, 107.

[32] W. W. Mullins, R. F. Sekerka, J. Appl. Phys. 1964, 35, 444.

[33] J. T. Lee, R. A. Brown, In MRS Proceedings; 1990; Vol. 205, p. 319. 


\section{WILEY-VCH}

[34] R. J. Braun, S. H. Davis, Acta Metall. Mater. 1992, 40, 2617.

[35] J. M. Warrender, J. Mathews, D. Recht, M. Smith, S. Gradečak, M. J. Aziz, J. Appl. Phys. 2014, $115,163516$.

[36] K. Ishida, T. Nishizawa, M. E. Schlesinger, J. Phase Equilibria 1991, 12.

[37] A. Horsfield, H. Fujitani, Phys. Rev. B 2001, 63.

[38] M. J. Aziz, W. J. Boettinger, Acta Metall. Mater. 1994, 42, 527.

[39] B. C. Larson, J. Z. Tischler, D. M. Mills, J. Mater. Res. 1986, 1, 144.

[40] J. A. Kittl, P. G. Sanders, M. J. Aziz, D. P. Brunco, M. O. Thompson, Acta Mater. 2000, 48, 4797.

[41] M. Amoorezaei, S. Gurevich, N. Provatas, Acta Mater. 2010, 58, 6115.

[42] L. K. Aagesen, A. E. Johnson, J. L. Fife, P. W. Voorhees, M. J. Miksis, S. O. Poulsen, E. M. Lauridsen, F. Marone, M. Stampanoni, Acta Mater. 2011, 59, 4922.

[43] L. K. Aagesen, A. E. Johnson, J. L. Fife, P. W. Voorhees, M. J. Miksis, S. O. Poulsen, E. M. Lauridsen, F. Marone, M. Stampanoni, Nat. Phys. 2010, 6, 796.

[44] G. J. van Gurp, G. E. J. Eggermont, Y. Tamminga, W. T. Stacy, J. R. M. Gijsbers, Appl. Phys. Lett. 1979, 35, 273.

[45] B. Gault, M. P. Moody, F. de Geuser, G. Tsafnat, A. La Fontaine, L. T. Stephenson, D. Haley, S. P. Ringer, J. Appl. Phys. 2009, 105, 034913. 